\title{
Development of a Motion Sensing and Automatic Positioning Universal Planisphere Using Augmented Reality Technology
}

\author{
Wernhuar Tarng, Jiong-Kai Pan, and Chiu-Pin Lin \\ Graduate Institute of e-Learning Technology, National Hsinchu University of Education, Hsinchu, Taiwan \\ Correspondence should be addressed to Wernhuar Tarng; wtarng@nhcue.edu.tw
}

Received 3 June 2016; Revised 9 December 2016; Accepted 26 December 2016; Published 14 February 2017

Academic Editor: Stefania Sardellitti

Copyright (C) 2017 Wernhuar Tarng et al. This is an open access article distributed under the Creative Commons Attribution License, which permits unrestricted use, distribution, and reproduction in any medium, provided the original work is properly cited.

\begin{abstract}
This study combines the augmented reality technology and the sensor functions of GPS, electronic compass, and 3-axis accelerometer on mobile devices to develop a motion sensing and automatic positioning universal planisphere. It can create local star charts according to the current date, time, and position and help users locate constellations on the planisphere easily through motion sensing operation. By holding the mobile device towards the target constellation in the sky, the azimuth and elevation angles are obtained automatically for mapping to its correct position on the star chart. The proposed system combines observational activities with physical operation and spatial cognition for developing correct astronomical concepts, thus making learning more effective. It contains a built-in 3D virtual starry sky to enable observation in classroom for supporting teaching applications. The learning process can be shortened by setting varying observation date, time, and latitude. Therefore, it is a useful tool for astronomy education.
\end{abstract}

\section{Introduction}

Astronomy is a natural science for studying celestial bodies in the universe, and its scope covers the entire astronomical objects and their interactions. Therefore, the earth and the solar system which we live in are also included. The changes of astronomical phenomena have great influences on our daily lives. Observing celestial bodies such as the sun, the moon, and stars is an essential part in astronomy education. By observing the astronomical phenomenon, students can investigate the relation between the movement of celestial bodies and their interactions and use scientific methods to solve problems for deriving answers, so it can enhance their critical thinking and problem solving skills.

Some astronomical phenomena such as sunrise, sunset, and the movement of stars in the sky can be seen in our daily lives and they are directly related to the rotation and revolution of the earth. Since the ancient times, human beings began to keep track of stars in the night sky to tell directions and seasonal changes. By connecting brighter stars with imaginary lines, the ancestors created a number of constellations and each constellation was named after its pattern and mythological story. In 1922, Russell aided the International Astronomical Union in dividing the celestial sphere into 88 official constellations [1]. To define the star's precise position in the sky, Lundmark at Lund Observatory led a group of draftsmen to complete the project of marking some 7000 stars on the celestial sphere, where the brightness of stars was represented by different sizes of white dots [2].

Observing stars is an important learning activity in astronomy education. However, it has to be conducted during night time, and the observation is easily affected by weather conditions or obstructed by surrounding high buildings. Without facilities such as the dome screen or simulation software like Stellarium (http://www.stellarium.org) and Google Sky Map (http://groups.google.com/group/google-sky-map), star observation can only be done in its natural way. That is, it has to be conducted outdoors at night according to the time and season of the star's appearance.

Stellarium is free desktop software which renders realistic skies in real time with Open Graphics Library (openGL) (https://www.opengl.org). With Stellarium, the user can see the visible stars in the sky with the naked eye, binoculars, or a small telescope. It can also be used in planetarium 
projectors by setting the user's coordinates. Google Sky Map is an Android version of Google Sky. The application enables the user to pinpoint the exact location of the stars, planets, and other celestial objects in the night sky. It can be used on a mobile device as an augmented reality application. When pointing the mobile device at the sky, the user can see details of 3D starry sky represented by a sky map.

Michie [3] and Orion [4] considered star observation in the night sky important and helpful for constructing astronomical concepts, but it is difficult in practice for teachers to conduct teaching activities during night time. Without sufficient observation and verification, students can only think about the models of earth and celestial sphere to explain the stars' movement in the sky due to the earth's rotation and thus some common misconceptions in astronomy may still exist [5-7].

The traditional planisphere has the advantages of light weight, portability, easy acquisition, and low price, but it also has some restrictions such as applicable areas, time, and visible stars. It is an astronomical observation tool developed by projecting the celestial sphere and its stars towards the north celestial pole to form a star chart (or sky map). As a result, the constellations shown on the star chart may often be distorted, especially those in the southern sky. To remedy this problem, most planispheres provide both the northern and the southern star charts on the front and the back sides for easy star observation.

When using a planisphere to find constellations in the night sky, the users must rotate the movable portion until the current date and time marked on its edge are aligned. The compass and protractor must also be used to measure the target constellation's azimuth and elevation angles. Since the equatorial coordinate system in the celestial sphere is different from the Cartesian coordinate system on the ground, students may have difficulty locating a constellation on the star chart or deciding when to switch to the northern or the southern star chart. In addition, the traditional planisphere is only applicable to a certain area (or latitude). If the observation is to be conducted in another area with a different latitude, we need to use the planisphere suitable for that area.

With the advance of information technology, computer software can be applied to simulate scientific phenomena such as physical or chemical reactions for observation in the microscopic world. It can also reduce the amount of experimental variables for learners to focus on specific subjects to enable conceptual change $[8,9]$. Nowadays, mobile devices such as the personal digital assistance (PDA), smartphone, and tablet PC have been integrated into different educational applications. As a result, learning activities are no longer restricted to the classroom. In other words, they can be done anytime and anywhere by using any device to achieve ubiquitous learning [10].

Recently, the hardware of mobile devices becomes more powerful, and the built-in sensors such as the GPS, electronic compass, and 3-axis accelerometer can provide the information of position, time, direction, acceleration, and so on to support the design of simulation software for applications in different areas of education. Schiller and Voisard [11] proposed the concept of context awareness by using the GPS to obtain the user's current location for providing immediate services. The main objective is to satisfy the sensational requirement by updating the information according to environmental changes such as local date, time, position, and direction.

Augmented reality (AR) is a view of the real world where elements are augmented by computer-generated situations to enhance the perception of reality. Its purpose is to incorporate virtual objects into the real world to enhance their interactions with the users. According to Azuma's [12] definition, $\mathrm{AR}$ is an evolution of virtual reality (VR) with the following features: (1) interacting with real and virtual environments, (2) providing real-time feedback, and (3) necessarily being in the $3 \mathrm{D}$ space. In comparison, VR is a technology to create an interactive environment for simulating the real world through one's sense organs. The users can see, hear, and feel in the created scenes as if situated in the real world and even interact with the objects in the virtual scenes [13]. AR integrates the real world with virtual objects to increase the sense of reality in a more interactive way, and it provides useful information not directly available to enhance one's comprehension in the real environments.

Liu et al. [14] introduced several AR systems with which students can view the virtual solar system on the classroom table and visualize the process of photosynthesis. Kerawalla et al. [15] combined the whiteboard, projector, web camera, AR technology, and 3D modeling package for students to learn about the earth and the sun as well as the changes of day and night. It was discovered in their study that teachers realized the advantages of using 3D images and believed that AR can make inaccessible subject matters available to students. Therefore, AR can increase learners' interaction with the real world and provide them with useful information for perceiving some scientific phenomena which cannot be experienced in the real world [10].

In this study, we have combined the AR technology and the sensor functions of GPS, electronic compass, and 3-axis accelerometer on mobile devices to develop a motion sensing and automatic positioning universal planisphere. It can create local star charts according to the current position, date, and time and help the user locate constellations on the star chart easily through motion sensing operation. By holding the mobile device towards the target constellation in the sky, the azimuth and elevation angles are mapped to the corresponding position on the star chart. With the function of searching constellations, the user can find the target constellation easily by following the instruction. By setting the observation date, time, and latitude, the user can see the change on the star chart to understand that constellations seen at different time, in different seasons, or at different latitudes are also different.

The system can shorten the learning process by changing the setting for observation. With the built-in virtual 3D starry sky, it can solve the problem of being unable to observe stars due to bad weather conditions or obstruction by surrounding high buildings. In addition, the physical operation can make a deeper impression on students, enabling them to store the acquired knowledge in long-term memory. The system combines observational activities with physical operation 

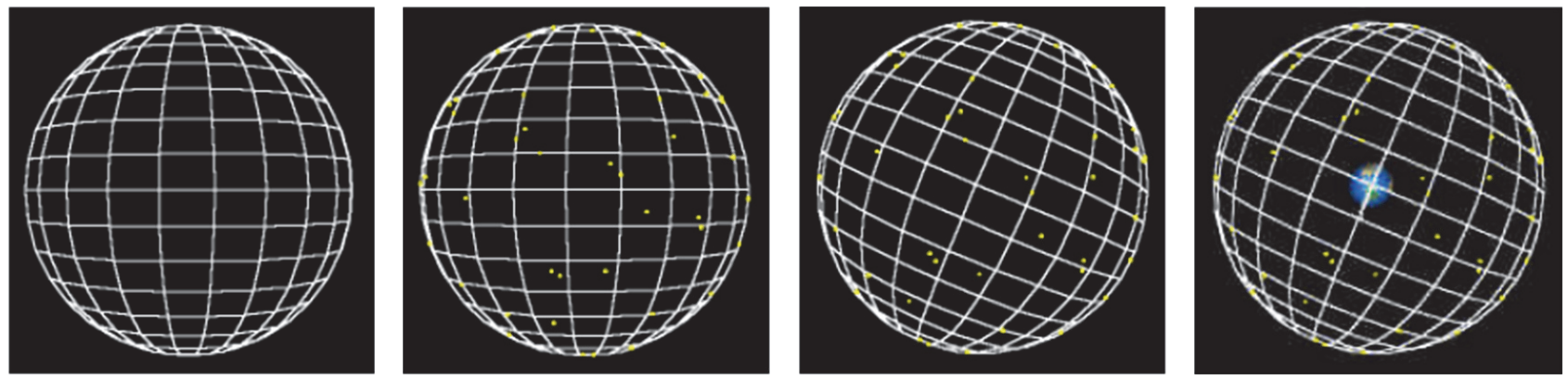

FIGURE 1: Developing the celestial sphere and earth model.

and spatial cognition for developing correct astronomical concepts. Therefore, it is a useful teaching aid and observation tool for astronomy education in elementary and high schools.

In this study, a teaching experiment has been conducted to investigate students' learning effectiveness by using the universal planisphere as a tool for star observation. The results are compared with those of using other tools. In addition, a questionnaire survey has been performed to analyze and compare the attitudes of students after using different observation tools, and the results could also be adopted as a reference for improving the system functions. The rest of this paper is organized as follows: Section 2 describes the system design, Section 3 provides the experimental results and data analysis, and Section 4 is the conclusion.

\section{System Design}

The objective of the proposed system is to improve traditional planispheres by providing the functions of motion sensing and automatic positioning so that students can learn to operate the planisphere easily and establish correct concepts in astronomical observation and spatial cognition. It is designed as a teaching tool for the learning unit of "Star Observation" in science and technology curriculums for elementary schools with the following learning objectives [16]:

(i) Learn to use the planisphere and know the patterns of constellations and their mythological stories.

(ii) Understand that stars are moving from east to west through observation.

(iii) Understand that the starry sky in different seasons is also different.

(iv) Learn to locate the North Star using the constellations Cassiopeia the Queen and the Big Dipper.

The system of universal planisphere is composed of seven modules, including date and time adjustment, visual angle control, star chart generation, star chart switching, constellation positioning, online test, and test results upload. Their functions are described briefly in the following:

(i) Date and time adjustment: the user can set different date and time for observing constellations. (ii) Visual angle control: the system provides zoom-in and zoom-out functions so that the user can see constellations and their information more clearly.

(iii) Star chart generation: the local star charts can be generated according to the GPS data (longitude and latitude) as well as the current date and time.

(iv) Star chart switching: the system can switch between the northern and the southern star charts according to the user's direction when holding the mobile device towards the sky.

(v) Constellation positioning: the system can obtain the azimuth and elevation angles of the target constellation for mapping to its corresponding position on the star chart automatically.

(vi) Online test: an online test is provided to assess if the user can operate the planisphere correctly to find the target constellation.

(vii) Test results upload: the user's test results can be uploaded to the server via wireless networks for the teacher to examine their learning achievement.

2.1. Celestial Sphere. Since the stars are very far away from the earth, if we can create an arbitrarily large and transparent sphere, called the celestial sphere, with its center overlapping with the earth center, the connection line between the earth center and the star will intersect with the celestial sphere. Hence, the longitude and latitude of the star can be marked at the intersection point on the celestial sphere (Figure 1).

In this study, the celestial sphere and constellations model (Figure 2) is developed to simulate the starry sky and to generate the star charts on the universal planisphere. After obtaining the longitude, latitude, date, and time from the GPS on the mobile device, the system can compute the normal vector at the observation point towards the celestial sphere. The marked stars on the celestial sphere above the observation point can be projected to the visual plane to simulate the starry sky seen from the earth. The celestial sphere and constellations are both static, and the phenomenon of star movement is due to the earth's rotation and revolution around the sun. Since the normal vector at the observation point changes as the earth rotates, the stars on the visual plane will also rotate around the north celestial pole. 


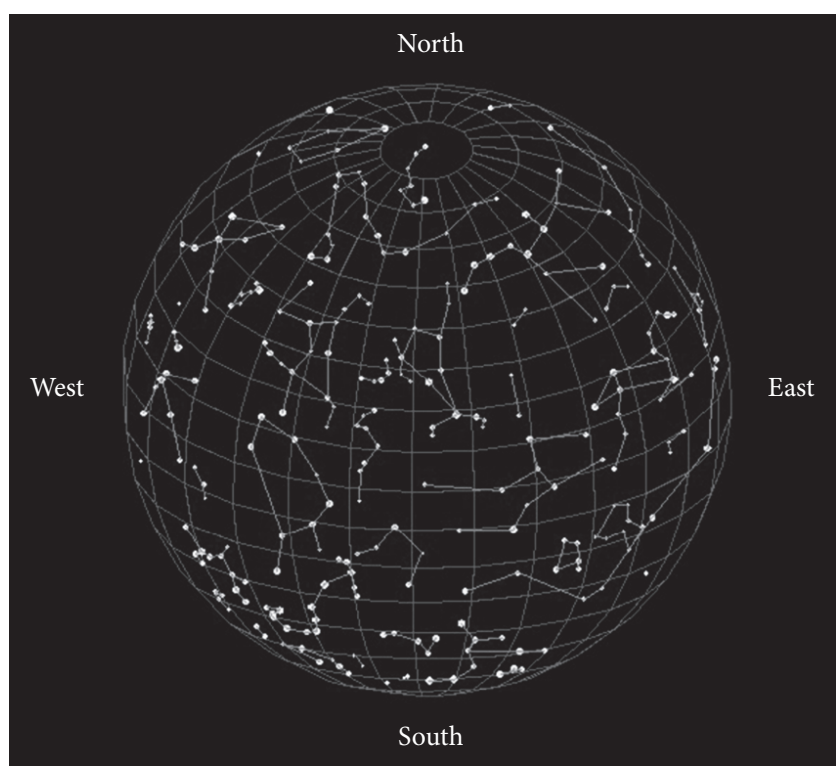

Figure 2: The celestial sphere and constellations model.

2.2. Star Chart. In order to design the universal planisphere applicable at different latitudes, the perspective projection is used to transform the $3 \mathrm{D}$ coordinates of constellations on the celestial sphere to the $2 \mathrm{D}$ coordinates on the star chart. First, rotate the earth and celestial sphere (including all constellations on it) so that the normal vector lands on the $x-z$ plane of the ground's 3D coordinate system. The ground's $3 \mathrm{D}$ coordinate system is a Cartesian coordinate system where $x$-axis points to the east and $y$-axis points to the north. Next, rotate the earth and the celestial sphere so that the normal vector at the observation point overlaps with the $z$-axis of the $3 \mathrm{D}$ coordinate system. The rotation matrixes for the $y$-axis and $z$-axis are specified in the following:

$$
\begin{aligned}
& {\left[R_{Y}(\beta)\right]=\left[\begin{array}{ccc}
\cos \beta & 0 & -\sin \beta \\
0 & 1 & 0 \\
\sin \beta & 0 & \cos \beta
\end{array}\right],} \\
& {\left[R_{Z}(\alpha)\right]=\left[\begin{array}{ccc}
\cos \alpha & \sin \alpha & 0 \\
-\sin \alpha & \cos \alpha & 0 \\
0 & 0 & 1
\end{array}\right] .}
\end{aligned}
$$

Finally, use the perspective projection method to map constellations on the celestial sphere to the $2 \mathrm{D}$ star chart according to the user's location (Figure 3 ). In Figure $3, \overrightarrow{\mathrm{SE}}$ is a space vector with length of one unit and its direction pointing from the sun to the earth and it can be used to compute the brightness of the sky. The formulas for perspective projection are given below:

$$
\begin{aligned}
& x^{\prime}=\frac{x}{1-z / Z_{V}}, \\
& y^{\prime}=\frac{y}{1-z / Z_{V}},
\end{aligned}
$$

where $Z_{V}$ is the observation point at the $Z$-axis.

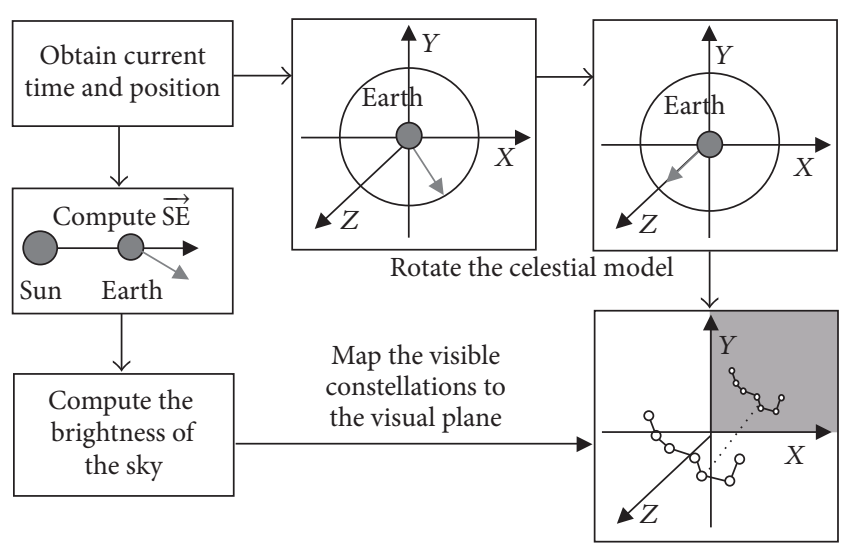

FIGURE 3: Mapping the constellations to create the star chart.

The process of generating the star chart is described in the following. First, the latitude at the user's location is obtained from the GPS as the offset angle from the north pole of celestial sphere. The star chart is generated using the perspective projection described above by rotating the celestial sphere along the $X$-axis for the offset angle. In addition, the system can use the data of constellations on the celestial sphere to create the virtual 3D starry sky and use the perspective projection to generate the northern and southern star charts for the application at the user's location. Figure 4 shows the star charts generated at different latitudes in the Northern Hemisphere.

2.3. Automatic Positioning. To provide the function of positioning constellations, the system first obtains the azimuth and elevation angles from the electronic compass and 3-axis accelerometer before applying the perspective projection to generate the star chart. The target constellation's position can also be located on the star chart using the same formulas, and it is marked with a red circle on the star chart for easy identification. The formulas for positioning constellations on the northern and southern star charts are shown in Table 1, where the target constellation's position in the original coordinate system (celestial sphere) is denoted by $(x, y, z)$, the local coordinate system (starry sky) is denoted by $\left(x^{\prime}, y^{\prime}, z^{\prime}\right)$, and the $2 \mathrm{D}$ coordinate system on the star chart is denoted by $\left(x^{\prime \prime}, y^{\prime \prime}\right)$. In addition, $\gamma$ stands for the offset angle (or latitude) where the user is located, and $\theta$ represents the azimuth angle of the target constellation.

According to the formulas described above, the module for mapping from the 3D coordinate system of the celestial sphere to the $2 \mathrm{D}$ coordinate system on the star chart can be developed. Figure 5 shows the $2 \mathrm{D}$ coordinate system on the star chart at the latitude of $23^{\circ} \mathrm{N}$. After rotating the movable portion of the planisphere to align the date and time, the azimuth and elevation angles of the target constellation can be obtained and mapped to the correct position on the star chart (marked by a red circle) for easy identification. When the user makes an observation by holding the mobile device towards the target constellation, the red circle will appear on the star chart to show its correct position. Upon locating 
TABLE 1: Formulas for positioning constellation on the universal planisphere.

\begin{tabular}{lcc}
\hline Original coordinate & Formulas for coordinate transformation & Local coordinate \\
\hline$x$ & $x^{\prime}=x$ & $x^{\prime}$ \\
$y$ & $y^{\prime}=y \times \cos (\pi \times \gamma / 180)+z \times \sin (\pi \times \gamma / 180)$ & $y^{\prime}$ \\
$z$ & $z^{\prime}=-y \times \sin (\pi \times \gamma / 180)+z \times \cos (\pi \times \gamma / 180)$ & $z^{\prime}$ \\
\hline & Formulas for mapping from 3D starry sky to 2D star chart & \\
\hline & $x^{\prime \prime}=\left(x^{\prime} / \sqrt{x^{\prime 2}+\left(y^{\prime}-y_{0}^{\prime}\right)^{2}}\right) \times$ ellipse_long $\times(90-\theta) / 90$ & \\
& $y^{\prime \prime}=\left(\left(y^{\prime}-y_{0}^{\prime}\right) / \sqrt{x^{\prime 2}+\left(y^{\prime}-y_{0}^{\prime}\right)^{2}}\right) \times$ ellipse_short $\times(90-\theta) / 90$ \\
\hline
\end{tabular}

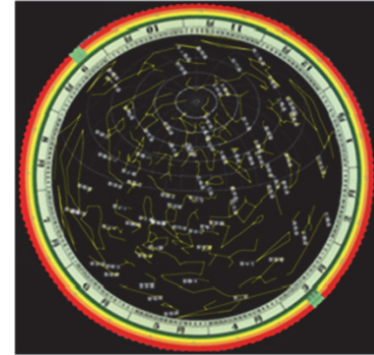

$15^{\circ}$

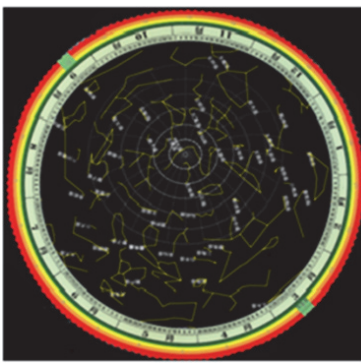

$60^{\circ}$

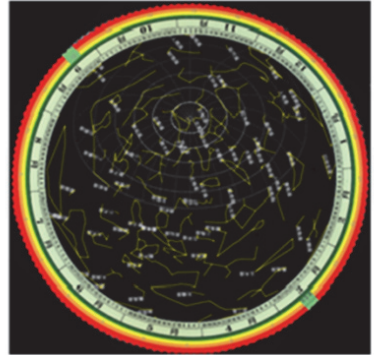

30

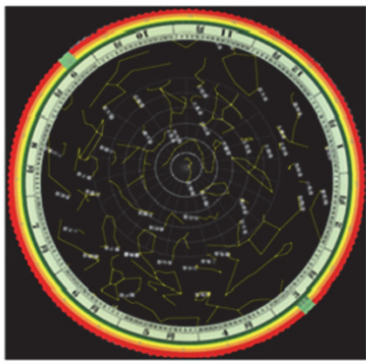

$75^{\circ}$

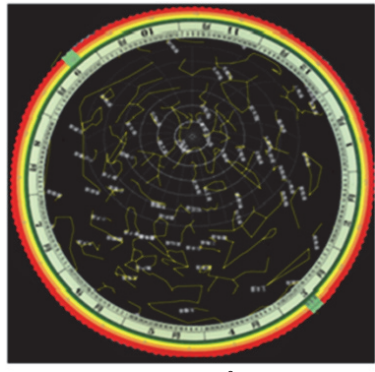

$45^{\circ}$

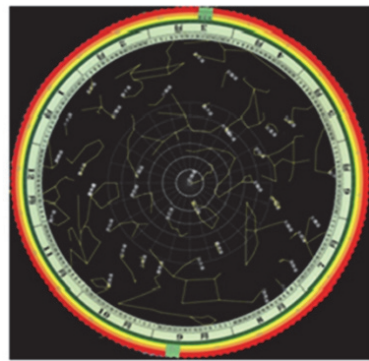

$90^{\circ}$

Figure 4: The star charts generated at different latitudes.

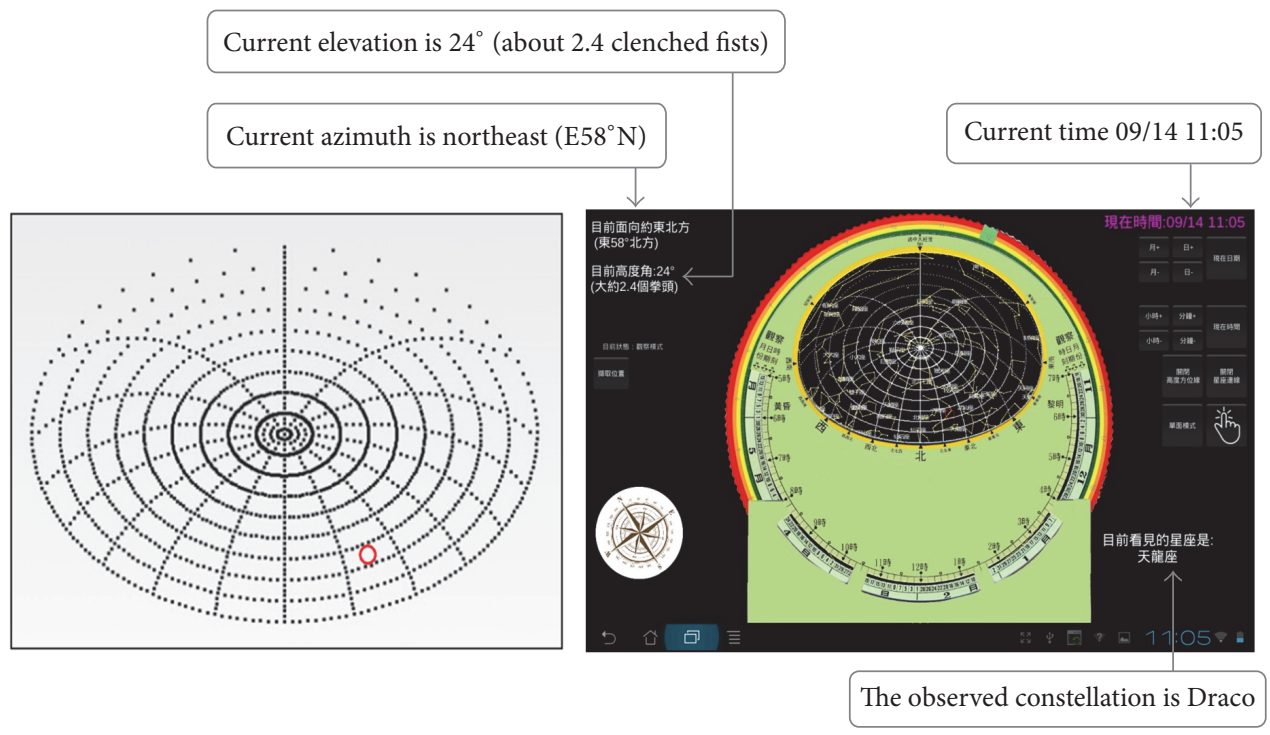

Figure 5: Mapping from 3D celestial sphere to 2D star chart. 


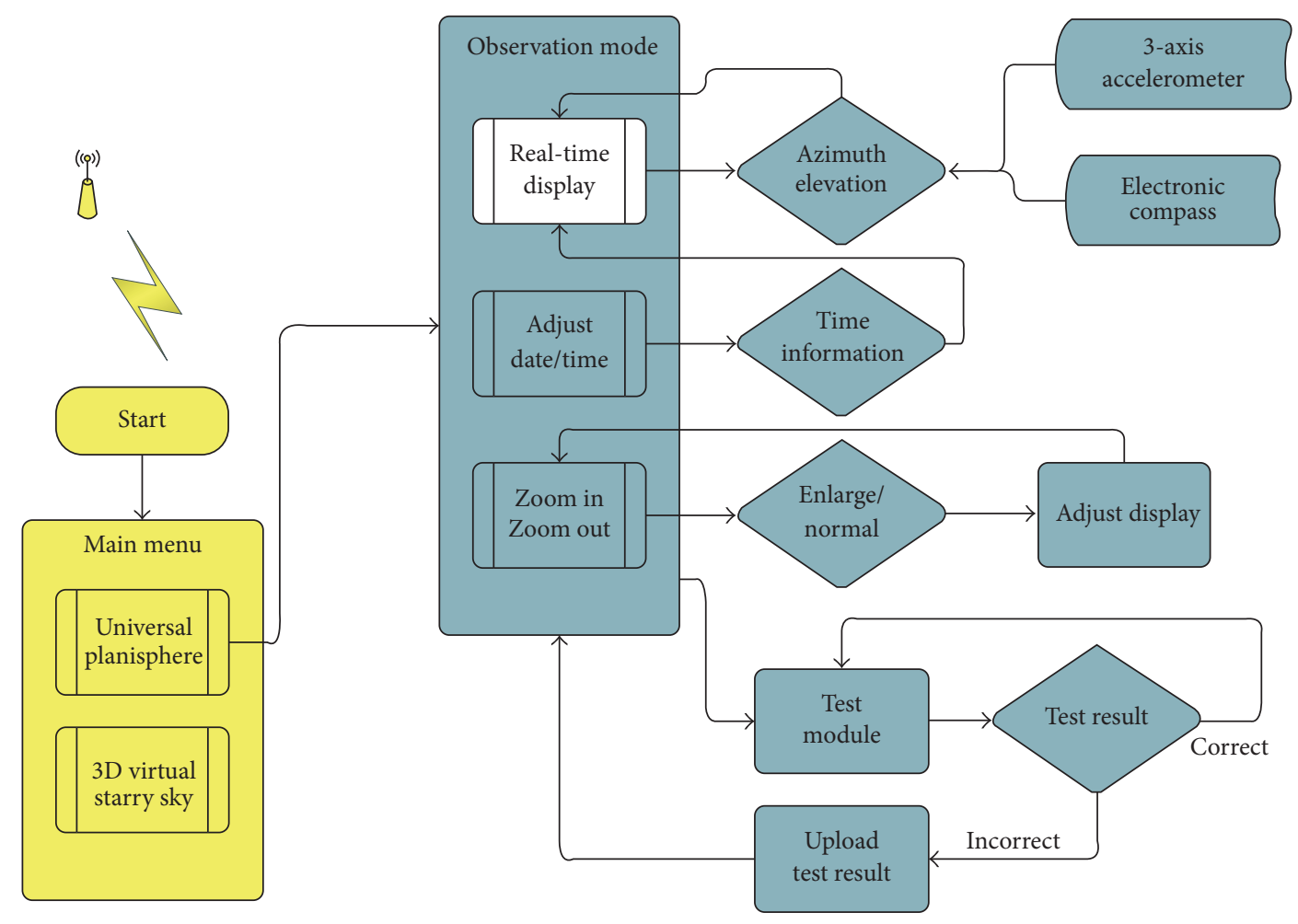

Figure 6: The flowchart of online test function.

the target constellation, the related data (constellation name, date, time, azimuth angle, and elevation angle) can be recorded by pressing the Record button. The recorded data can be uploaded for the teacher to check if the students have completed the observational assignment correctly.

2.4. Automatic versus Manual Operation. The prior knowledge required for conducting star observation is being able to use a planisphere to find the target constellation. Basically, the user has to rotate the movable portion to align the current date and time for the correct star chart to be shown on the planisphere. The adjustment is done manually on paper planispheres, and it can be done automatically on the universal planisphere. When doing so, the system simply obtains the current date and time through the Application Programming Interface (API) and converts them into the angle for rotating the movable portion of the planisphere. This study used the C\# programming language in Unity3D to develop the manual and automatic operation functions, which can be used to adjust the planisphere to show the star charts for different seasons (spring, summer, fall, and winter) and different time (evening, midnight, and dawn).

2.5. Online Test. The online test function is designed to provide the users with a formational evaluation. Therefore, they can conduct observation using the universal planisphere and take the online test to make sure whether they have learned the correct operational skills. With the automatic setting mode, the system adjusts the date and time automatically for the users to observe constellation easily, but they may not know how to operate a paper planisphere when required to do so. Hence, the system also provides the manual operation mode such that the users have the chance to perform the alignment of date and time. Using the online test function, the teacher can check if students have learned to adjust the planisphere and they can measure the azimuth and elevation angles of the target constellation to locate it on the star chart.

The flowchart of system operation is shown in Figure 6. When the user clicks the Test button on the screen, the test questions will appear at the lower part of the screen. To prevent the test question from blocking other information on the screen, the background of the test question is designed as semitransparent such that the user can also see the information behind it (Figure 7). After reading the test question, the user can provide the answer by clicking the Confirmation button. Then, the user will receive a message to verify if the answer is correct or not.

2.6. Star Chart Switching. As soon as the user starts the system, the sensors will detect the user's location, direction, and the current date and time to generate the star chart. In order to simulate the starry sky for astronomical observation, the background color is set to black on the screen. The digital compass and its related information are displayed at the lower left corner of the screen to inform the user of his or her direction. The current date and time are shown at the upper right corner, together with the functions of their adjustment. The star chart is situated at the center, taking most of the space, while the screen also displays some other information such as the position (longitude and latitude) and the direction 


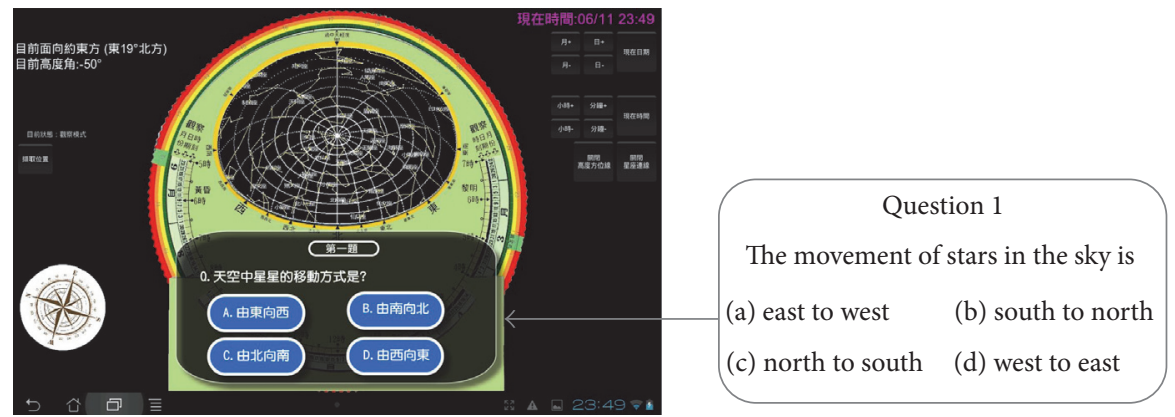

FIGURE 7: The test question on the universal planisphere.

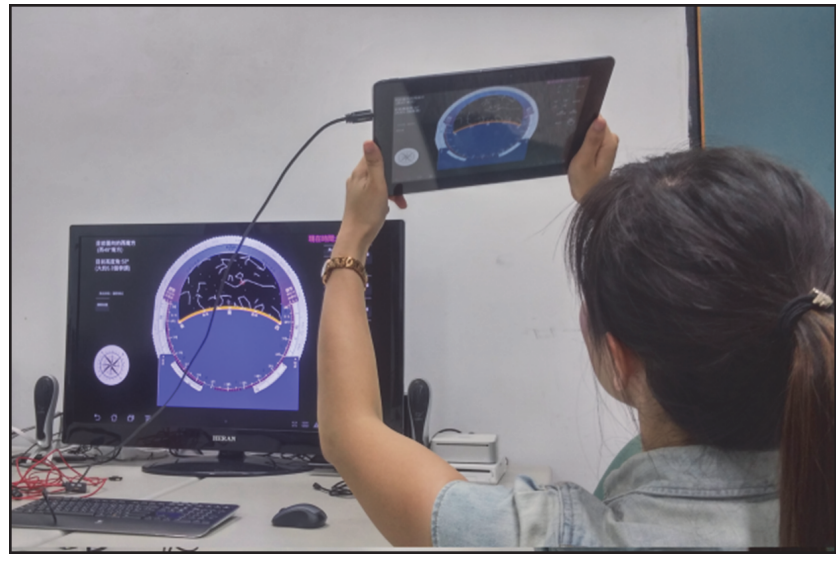

FIGURE 8: The universal planisphere switching to the southern star chart.

(azimuth and elevation angles). When the user turns to face the southern sky, the planisphere will display the southern star chart accordingly for the user to conduct observation in the opposite direction (Figure 8).

\section{Teaching Experiment}

A teaching experiment has been conducted to evaluate the learning effectiveness of students using the universal planisphere for star observation. The results are compared with those of using different tools (the Google Sky Map and paper planisphere). This study randomly selected three classes of fifth-grade students from an elementary school in Taichung, Taiwan, as the experimental samples to form three groups, that is, the experimental Group A (23 students), the experimental Group B (25 students), and the control Group C (23 students). According to experimental design, different observational tools were used by the three groups, respectively. Group A used the universal planisphere, Group B used the Google Sky Map, and Group C used the paper planisphere for classroom teaching and star observation after school. A questionnaire survey was also conducted to investigate the attitudes of students after using their tools for star observation. The variables of the teaching experiment are listed in Table 2.
TABLE 2: Variables in the teaching experiment.

\begin{tabular}{cl}
\hline Independent variables & \\
Group A & Universal planisphere \\
Group B & Google Sky Map \\
Group C & Paper planisphere \\
Covariance & Pretest \\
Dependent variables & Posttest, delayed posttest \\
Control variables & Teacher, teaching time, learning contents \\
\hline
\end{tabular}

The teaching experiment was conducted by following the guidelines of the "Astronomical Observation" learning unit in the K9 Science and Life Technology Curriculum for elementary and high schools in Taiwan [17]. This study adopted a nonequivalent pretest-posttest design involving different groups to investigate if significant differences exist among their learning achievements. Before the teaching activities, all students had taken the pretest for evaluating background knowledge in star observation, followed by the teaching activity using different tools for three weeks. After that, the posttest and questionnaire survey were conducted, and the delayed posttest was taken one month later (Figure 9). The achievement test and questionnaire results were collected for analyzing the learning effectiveness and attitudes of students after using different tools.

3.1. Background Knowledge. According to the background knowledge analysis (Figure 10), $62 \%$ of the students had the experience of star observation before the teaching experiment. The percentages of students with such an experience in the three groups are 52\% (Group A), 64\% (Group B), and 70\% (Group C), respectively. A further investigation shows that about one half of the experienced students had conducted star observation within the last 6 months (Figure 11).

3.2. Learning Effectiveness. The pretest was taken by the three groups of students one week before the teaching activity, and its analytical results by the ANOVA show that $F=0.08$ and $P=0.92>0.05$, indicating no significant difference in the background knowledge among three groups. After the teaching activity, the posttest was taken by the three groups for the investigation of their learning achievements. Analytical results by the paired sample $t$-test according to 
TABLE 3: Results of the paired sample $t$-test for Group A.

\begin{tabular}{lcccc}
\hline Test & Average & Student number & Standard deviation & $T$ \\
\hline Pretest & 50.00 & 23 & 16.10 & -7.47 \\
Posttest & 75.22 & 23 & 21.08 & $<0.001^{* * *}$ \\
\hline
\end{tabular}

${ }^{* * *} P$ value less than 0.001 .

TABLE 4: Results of the paired sample $t$-test for Group B.

\begin{tabular}{|c|c|c|c|c|c|}
\hline Test & Average & Student number & Standard deviation & $T$ & Significance \\
\hline Pretest & 51.60 & 25 & 18.86 & \multirow{2}{*}{-8.34} & \multirow{2}{*}{$<0.001^{* * *}$} \\
\hline Posttest & 74.20 & 25 & 13.98 & & \\
\hline
\end{tabular}

${ }^{* * *} P$ value less than 0.001 .

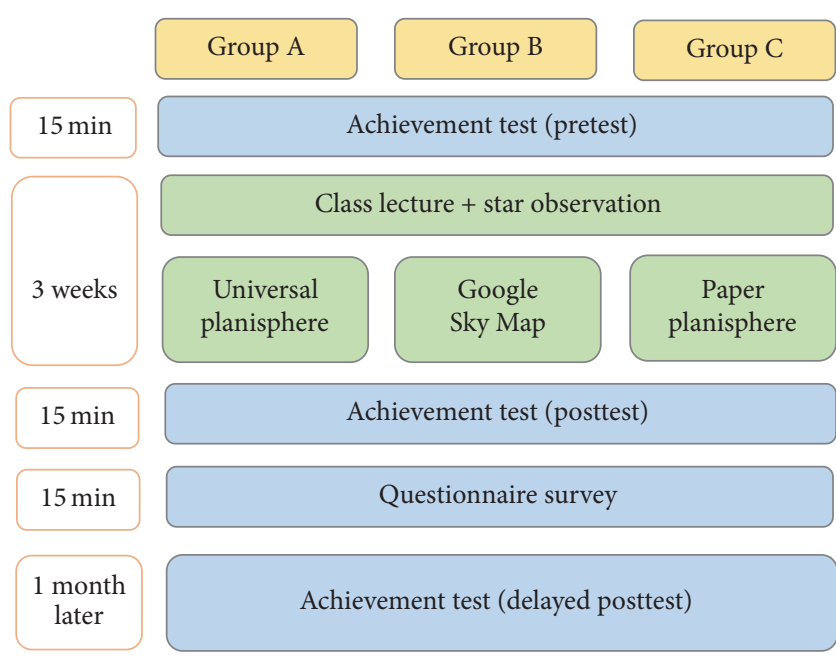

FIGURE 9: The flowchart of teaching experiment by three groups.

the pretest and posttest have shown significant improvement in learning effectiveness for Group A (Table 3), Group B (Table 4), and Group C (Table 5); that is, the universal planisphere developed in this study can enhance students' learning effectiveness in star observation, and so can the Google Sky Map and paper planisphere. After that, the ANOVA was conducted according to the pretest and posttest results of the three groups, and the analytical results have shown that $F=2.16$ and $P=0.12>0.05$, indicating no significant difference among the three groups.

This study also evaluated the operational skills of students in using their tools to conduct star observation. According to the statistical results in Table 6, students in Group A performed much better than those in Group B because the latter had difficulty in acquiring precise azimuth and elevation angles for locating the target constellations. Therefore, the universal planisphere can enhance students' operational skills in star observation. The average score of Group A is also higher than that of Group $\mathrm{C}$ but it has not achieved the standard of significant difference.

One month after the teaching activity, the delayed posttest was taken by the three groups for the investigation of their long-term learning achievements. Analytical results by the paired sample $t$-test show no significant difference between the posttest and delayed posttest for Group A (Table 7), Group B (Table 8), and Group C (Table 9), indicating the tools used by the three groups are effective in maintaining the learning achievement.

Considering the background knowledge of experimental samples may be different, a quasi-experimental approach using the pretest, posttest, and delayed posttest design for nonequivalent groups is adopted in this study to evaluate the learning effectiveness of students in different groups. Although the average score of Group A (Table 7) is lower than that of Group C (Table 9), the difference between the delayed posttest and posttest for Group A (5.43) is higher than that of Group C (-1.09). Therefore, the long-term learning effectiveness (or learning retention) of using the universal planisphere is better than that of using the paper planisphere for classroom teaching and star observation.

3.3. Learning Attitudes. The questionnaire survey used in this study is a system satisfaction evaluation according to the users' experiences in using their tools and it was designed after discussing with two experts in astronomy education and a science teacher. The questionnaire contains 20 questions which are divided into four sections (5 questions in learning contents, 4 questions in system functions and interface design, 5 questions in learning experience, and 6 questions in students' willingness of usage). This study adopted Likert's 5-point scale [18] (5 = strongly agree, $4=$ agree, $3=$ neutral, $2=$ disagree, and $1=$ strongly disagree) to measure the attitudes of students after using the tools. The validity of the questionnaire is ensured because the questions were reviewed and modified by the teacher and experts to enhance its correctness before performing the questionnaire survey. When preparing the questionnaire, it was first designed as an editable form for the teacher and experts to provide their suggestions to establish the content validity. We revised and deleted the inappropriate questions based on their opinions and then performed the questionnaire survey. The statistical software SPSS is used to perform the reliability analysis and the value of Cronbach's alpha is $0.85,0.95$, and 0.88 for Group A, Group B, and Group C, respectively, showing the results have met the standard of high reliability.

This study performed a statistical analysis on the questionnaire results by the three groups as the system satisfaction survey. The average score (Avg.) and standard deviation (SD) 
TABLE 5: Results of the paired sample $t$-test for Group C.

\begin{tabular}{lccccc}
\hline Test & Average & Student number & Standard deviation & $T$ & Significance \\
\hline Pretest & 51.96 & 23 & 17.43 & -7.80 & $<0.001^{* * *}$ \\
Posttest & 83.48 & 23 & 14.49 & \\
\hline
\end{tabular}

${ }^{* * *} P$ value less than 0.001 .

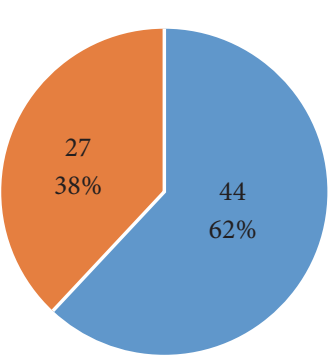

All students

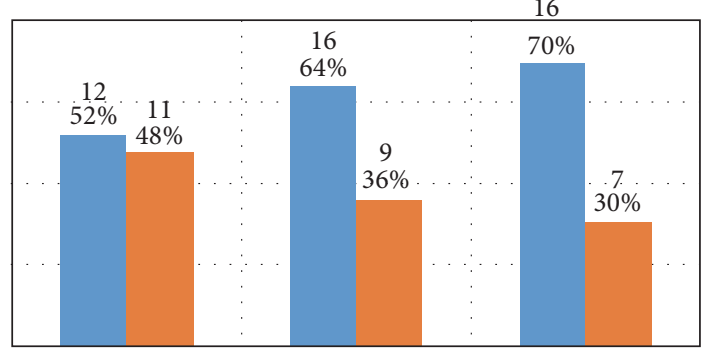

Group A
Group C

Experience in star observation $\quad$ Yes

No

FIGURE 10: The percentages of students with experience of star observation.

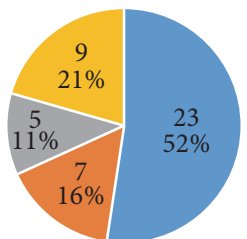

Less than 6 months

- 6 months to 1 year

1 year to 2 years

More than 2 years

Experienced students

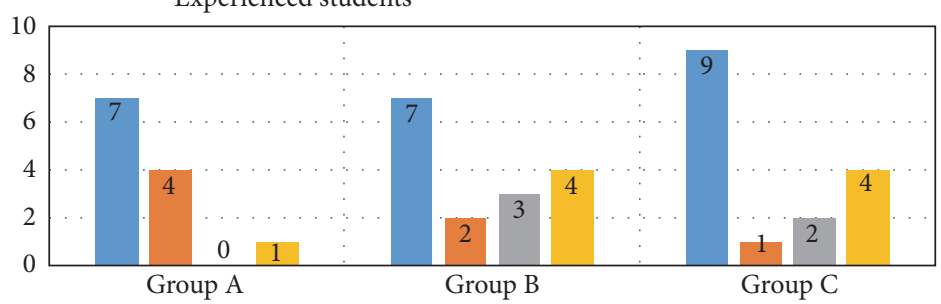

FIGURE 11: Students' experience of star observation within the last two years.

of each selected item are calculated for each question to further understand the attitudes of students after using their tools in star observation, and the statistical results are shown in Table 10. The ANOVA is also conducted to find out the difference of students' attitudes in each section and each question by the three groups, where the question results with high significance $(P<0.01)$ are highlighted with italic font. Also, the means of the average score and standard deviation for all questions are also calculated and listed at the bottom. The teacher and some students have provided feedback which can be used as reference for improving the system.

According to the questionnaire results, the average score by Group A is 4.67, which is higher than that of Group B (4.07) and Group C (4.24). The results show that the universal planisphere can enhance students' learning motivation and interest in star observation and it can satisfy the requirement of class teaching and outdoor star observation better than the other two tools. However, the average score by Group B is lower than that of Group C, indicating that students may have difficulty using the $3 \mathrm{D}$ tool in finding constellations and obtaining the required information. The ANOVA results show a significant difference among the three groups on learning contents (Table 11), interface design (Table 12), learning experiences (Table 13), and willingness of usage (Table 14).

In Table 11, the average score of Group A (4.75) is higher than that of Group C (4.40) and Group B (3.99). In Table 12, the average score of Group A (4.59) is higher than that of Group C (4.34) and Group B (4.02). In Table 13, the average score of Group A (4.68) is higher than that of Group C (4.26) 
TABLE 6: The statistical results of operational skills.

\begin{tabular}{|c|c|c|c|c|c|}
\hline Group & Student number & Average & Standard deviation & $F$ & Significance \\
\hline $\mathrm{A}$ & 23 & 91.74 & 7.01 & \multirow{3}{*}{17.02} & \multirow{3}{*}{$<0.001^{* * *}$} \\
\hline B & 25 & 80.40 & 6.93 & & \\
\hline $\mathrm{C}$ & 23 & 89.13 & 7.33 & & \\
\hline
\end{tabular}

TABLE 7: Results of the paired sample $t$-test for Group A.

\begin{tabular}{|c|c|c|c|c|c|}
\hline Test & Average & Student number & Standard deviation & $T$ & Significance \\
\hline Posttest & 75.22 & 23 & 21.08 & \multirow{2}{*}{-1.641} & \multirow{2}{*}{$0.12>0.05$} \\
\hline Delayed posttest & 80.65 & 23 & 13.51 & & \\
\hline
\end{tabular}

TABLE 8: Results of the paired sample $t$-test for Group B.

\begin{tabular}{|c|c|c|c|c|c|}
\hline Test & Average & Student number & Standard deviation & $T$ & $\overline{\text { Significance }}$ \\
\hline Posttest & 74.20 & 25 & 13.97 & \multirow{2}{*}{-1.66} & \multirow{2}{*}{$0.11>0.05$} \\
\hline Delayed posttest & 77.20 & 25 & 12.00 & & \\
\hline
\end{tabular}

TABLE 9: Results of the paired sample $t$-test for Group C.

\begin{tabular}{lccccc}
\hline Test & Average & Student number & Standard deviation & $T$ & Significance \\
\hline Posttest & 83.48 & 23 & 14.49 & 0.89 & $0.38>0.05$ \\
Delayed posttest & 82.39 & 23 & 10.75 & \\
\hline
\end{tabular}

and Group B (4.14). In Table 14, the average score of Group A (4.75) is higher than that of Group B (4.10) and Group C (3.96), all significantly.

The interview records from the teacher and students after the experiment are summarized in the following to support the validity of the questionnaire results.

(1) Most students considered the system very convenient to use because they could find the location of a star easily without looking at the compass.

(2) The function of automatic positioning is helpful and it can reduce the time of identifying the star location on the star chart.

(3) Automatic mapping of elevation angle on the star chart can solve the problem of the method taught in the textbook by raising one's hand to estimate the elevation angle for which it is not very accurate.

(4) The system can be used in classroom to reduce the time of teaching students how to use the planisphere to enhance the skills in star observation.

\section{Conclusions}

Astronomical observation is an important subject in science and technology curriculums in elementary and high schools. By observing astronomical phenomena and using scientific methods to solve the problems, students can acquire correct answers and enhance their critical thinking and problem solving skills. This study combined the augmented reality technology and sensor functions of GPS, electronic compass, and 3-axis accelerometer on mobile devices to develop a motion sensing and automatic positioning universal planisphere. It can generate the local star chart according to the user's current position, date, and time. By holding the mobile device towards the target constellation in the sky, its azimuth and elevation angles can be obtained and mapped to the corresponding position on the star chart. The user can find the target constellation by following the system instruction easily. It also contains a built-in $3 \mathrm{D}$ virtual starry sky so that the observation can be done in the classroom during daytime for teaching applications.

A teaching experiment has been conducted to evaluate students' learning effectiveness after using the universal planisphere for star observation. The experimental results indicate that there is no significant difference among the three groups of students in their learning achievements, showing that the universal planisphere and the other tools were effective in maintaining the learning achievement. However, students' observational skills as well as their learning achievements have been improved significantly when using the universal planisphere. The questionnaire results reveal that most students considered the operation of universal planisphere and its user interface easy to use. Also, it can help them locate the target constellations quickly and made the learning process more interesting. 
TABLE 10: The questionnaire results for the three groups.

\begin{tabular}{|c|c|c|c|c|c|c|c|}
\hline & \multirow{2}{*}{ Evaluation questions } & \multicolumn{2}{|c|}{ Group A } & \multicolumn{2}{|c|}{ Group B } & \multicolumn{2}{|c|}{ Group C } \\
\hline & & Avg. & SD & Avg. & $\mathrm{SD}$ & Avg. & $\mathrm{SD}$ \\
\hline \multirow{5}{*}{ Learning contents } & $\begin{array}{l}\text { (1) The tool enables me to find the target } \\
\text { constellation's azimuth angle quickly. }\end{array}$ & 4.57 & 0.84 & 4.24 & 0.88 & 4.39 & 0.72 \\
\hline & $\begin{array}{l}\text { (2) The tool enables me to find the target } \\
\text { constellation's elevation angle quickly. }\end{array}$ & 4.70 & 0.70 & 3.72 & 0.94 & 4.22 & 1.00 \\
\hline & $\begin{array}{l}\text { (3) The tool enables me to verify which } \\
\text { constellation a star belongs to quickly. }\end{array}$ & 4.83 & 0.49 & 4.20 & 0.71 & 4.61 & 0.66 \\
\hline & $\begin{array}{l}\text { (4) Using the tool lets me know that the } \\
\text { North Star is static in the sky. }\end{array}$ & 4.74 & 0.54 & 3.96 & 0.98 & 4.70 & 0.56 \\
\hline & $\begin{array}{l}\text { (5) Using the tool lets me know that the } \\
\text { stars are rotating from the east to the west. }\end{array}$ & 4.91 & 0.42 & 3.84 & 0.75 & 4.52 & 0.73 \\
\hline \multirow{4}{*}{ Interface design } & (6) The tool is easy to operate. & 4.17 & 0.89 & 4.12 & 0.97 & 4.43 & 1.03 \\
\hline & (7) Operating the tool is interesting. & 4.83 & 0.39 & 4.08 & 0.81 & 4.17 & 1.11 \\
\hline & $\begin{array}{l}\text { (8) The tool helps me find the constellations } \\
\text { quickly during outdoor observation. }\end{array}$ & 4.65 & 0.83 & 3.96 & 0.84 & 4.52 & 0.73 \\
\hline & $\begin{array}{l}\text { (9) The information on the operation } \\
\text { interface is easy to understand. }\end{array}$ & 4.70 & 0.64 & 3.92 & 0.91 & 4.22 & 1.04 \\
\hline \multirow{5}{*}{ Learning experience } & $\begin{array}{l}\text { (10) The tool makes the learning unit of } \\
\text { star observation more interesting. }\end{array}$ & 4.74 & 0.54 & 4.24 & 0.78 & 4.35 & 0.71 \\
\hline & $\begin{array}{l}\text { (11) The tool that I am using is more } \\
\text { helpful than the tools used by the other } \\
\text { groups. }\end{array}$ & 4.78 & 0.42 & 4.36 & 0.70 & 4.13 & 0.97 \\
\hline & $\begin{array}{l}\text { (12) The tool gives me a deeper impression } \\
\text { on the learning contents. }\end{array}$ & 4.87 & 0.46 & 4.12 & 0.78 & 4.57 & 0.59 \\
\hline & $\begin{array}{l}\text { (13) The tool helps me complete recording } \\
\text { of star observation. }\end{array}$ & 4.57 & 0.79 & 4.12 & 0.93 & 4.48 & 0.59 \\
\hline & $\begin{array}{l}\text { (14) The tool can increase my motivation } \\
\text { in outdoor star observation. }\end{array}$ & 4.43 & 0.73 & 3.88 & 0.97 & 3.78 & 1.04 \\
\hline \multirow{7}{*}{ Willingness of usage } & $\begin{array}{l}\text { (15) It is very convenient to use the tool for } \\
\text { outdoor star observation. }\end{array}$ & 4.87 & 0.46 & 4.08 & 0.81 & 2.74 & 1.63 \\
\hline & $\begin{array}{l}\text { (16) I like to use the tool for star } \\
\text { observation. }\end{array}$ & 4.70 & 0.77 & 4.32 & 0.80 & 3.91 & 1.38 \\
\hline & $\begin{array}{l}\text { (17) I will continue using the tool for star } \\
\text { observation in the future. }\end{array}$ & 4.83 & 0.49 & 4.04 & 0.89 & 4.26 & 1.05 \\
\hline & $\begin{array}{l}\text { (18) I will share the experience of using } \\
\text { the tool with my classmates. }\end{array}$ & 4.48 & 0.95 & 3.92 & 1.04 & 4.13 & 1.18 \\
\hline & $\begin{array}{l}\text { (19) I will recommend the tool to my } \\
\text { family and friends in conducting star } \\
\text { observation. }\end{array}$ & 4.65 & 0.89 & 3.80 & 1.00 & 4.13 & 1.10 \\
\hline & $\begin{array}{l}\text { (20) On the whole, I am satisfied with the } \\
\text { usage of the tool. }\end{array}$ & 4.96 & 0.21 & 4.44 & 0.82 & 4.61 & 0.84 \\
\hline & Overall & 4.67 & 0.62 & 4.07 & 0.87 & 4.24 & 0.93 \\
\hline
\end{tabular}

TABLE 11: The ANOVA results of students' attitude on learning contents.

\begin{tabular}{|c|c|c|c|c|c|}
\hline Group & Student number & Average & Standard deviation & $F$ & Significance \\
\hline A & 23 & 4.75 & 0.44 & \multirow{3}{*}{12.12} & \multirow{3}{*}{$<0.001^{* * *}$} \\
\hline B & 25 & 3.99 & 0.58 & & \\
\hline $\mathrm{C}$ & 23 & 4.40 & 0.59 & & \\
\hline
\end{tabular}


TABLE 12: The ANOVA results of students' attitude on interface design.

\begin{tabular}{lccccc}
\hline Group & Student number & Average & Standard deviation & $F$ & Significance \\
\hline A & 23 & 4.59 & 0.47 & 4.42 & $<0.05^{*}$ \\
B & 25 & 4.02 & 0.70 & 0.78 & \\
C & 23 & 4.34 & &
\end{tabular}

TABLE 13: The ANOVA results of students' attitude on learning experiences.

\begin{tabular}{|c|c|c|c|c|c|}
\hline Group & Student number & Average & Standard deviation & $F$ & Significance \\
\hline $\mathrm{A}$ & 23 & 4.68 & 0.41 & & \\
\hline $\mathrm{B}$ & 25 & 4.14 & 0.70 & 6.00 & $<0.01^{* *}$ \\
\hline $\mathrm{C}$ & 23 & 4.26 & 0.51 & & \\
\hline
\end{tabular}

${ }^{* *} P$ value less than 0.01 .

TABLE 14: The ANOVA results of students' attitude on willingness of usage.

\begin{tabular}{|c|c|c|c|c|c|}
\hline Group & Student number & Average & Standard deviation & $F$ & Significance \\
\hline A & 23 & 4.75 & 0.34 & \multirow{3}{*}{9.35} & \multirow{3}{*}{$<0.001^{* * *}$} \\
\hline B & 25 & 4.10 & 0.72 & & \\
\hline $\mathrm{C}$ & 23 & 3.96 & 0.82 & & \\
\hline
\end{tabular}

The universal planisphere developed in this study provides astronomical learning contents which can be used anytime and anywhere. It converts observational activities into organized and meaningful knowledge and combines spatial cognition with the establishment of correct astronomical knowledge and concepts. The functions of mapping the azimuth and elevation angles of the target constellation in the sky to its corresponding position on the star chart as well as switching between the northern and southern star charts can help students establish correct directional and spatial concepts through physical operation. By using the universal planisphere for long-term observation, they can understand the relation between the earth's rotation and the change of astronomical phenomena. The application of universal planisphere is not limited by weather conditions or the obstruction of surrounding high buildings. Besides, the learning process can be shortened by setting different observation time and locations to reduce the workload of teachers and students. Therefore, it is a useful tool for astronomy education in elementary and high schools.

\section{Competing Interests}

The authors declare that there are no competing interests regarding the publication of this paper.

\section{Acknowledgments}

The authors wish to acknowledge the support by the Ministry of Science and Technology (MOST), Taiwan, under Contract nos. 103-2511-S-134-007-MY2 and 105-2514-S-134-003.

\section{References}

[1] National Audubon Society, Field Guide to the Night Sky, Alfred A. Knopf, Inc, New York, NY, USA, 1991.

[2] E. Chaisson and S. McMillan, Astronomy: A Beginner's Guide to the Universe, Pearson, Boston, Mass, USA, 2013.

[3] M. Michie, "Factors influencing secondary science teachers to organize and conduct field trips," Australian Science Teachers' Journal, vol. 44, no. 4, pp. 43-50, 1998.

[4] N. Orion, "A model for the development and implementation of field trips as an integral part of the science curriculum," School Science and Mathematics, vol. 93, no. 6, pp. 325-331, 1993.

[5] K. J. Schoon, "Students' alternative conceptions of Earth and space," Journal of Geological Education, vol. 40, no. 3, pp. 209214, 1992.

[6] J. Baxter, "Children's understanding of familiar astronomical events," International Journal of Science Education, vol. 11, no. 5, pp. 502-513, 1989.

[7] K. C. Trundle and R. L. Bell, "The use of a computer simulation to promote conceptual change: A Quasi-experimental Study," Computers and Education, vol. 54, no. 4, pp. 1078-1088, 2010.

[8] T. De Jong and W. R. Van Joolingen, "Scientific discovery learning with computer simulations of conceptual domains," Review of Educational Research, vol. 68, no. 2, pp. 179-201, 1998.

[9] W. Winn, F. Stahr, C. Sarason, R. Fruland, P. Oppenheimer, and Y.-L. Lee, "Learning oceanography from a computer simulation compared with direct experience at sea," Journal of Research in Science Teaching, vol. 43, no. 1, pp. 25-42, 2006.

[10] P.-H. Wu, G.-J. Hwang, L.-H. Su, and Y.-M. Huang, "A contextaware mobile learning system for supporting cognitive apprenticeships in nursing skills training," Educational Technology \& Society, vol. 15, no. 1, pp. 223-236, 2012. 
[11] J. Schiller and A. Voisard, Location-Based Services, Morgan Kaufmann, San Francisco, Calif, USA, 2004.

[12] R. T. Azuma, "A survey of augmented reality," Presence: Teleoperators and Virtual Environments, vol. 6, no. 4, pp. 355-385, 1997.

[13] G. Kim, Designing Virtual Reality Systems: The Structured Approach, Springer, Heidelberg, Germany, 2005.

[14] W. Liu, A. D. Cheok, C. L. Mei-Ling, and Y.-L. Theng, "Mixed reality classroom-learning from entertainment," in Proceedings of the 2nd International Conference on Digital Interactive Media in Entertainment and Arts (DIMEA '07), pp. 65-72, ACM, Perth, Australia, September 2007.

[15] L. Kerawalla, R. Luckin, S. Seljeflot, and A. Woolard, “Making it real': exploring the potential of augmented reality for teaching primary school science," Virtual Reality, vol. 10, no. 3, pp. 163174, 2006.

[16] Kang Hsuan Educational Publishing Group, 2014, https://www .knsh.com.tw/Index.asp.

[17] Ministry of Education, K9 Science and Life Technology Curriculum Standards, Ministry of Education, Ministry of Education, Taipei, Taiwan, 2012.

[18] R. Likert, "A technique for the measurement of attitudes," Archives of Psychology, vol. 22, no. 140, pp. 1-55, 1932. 

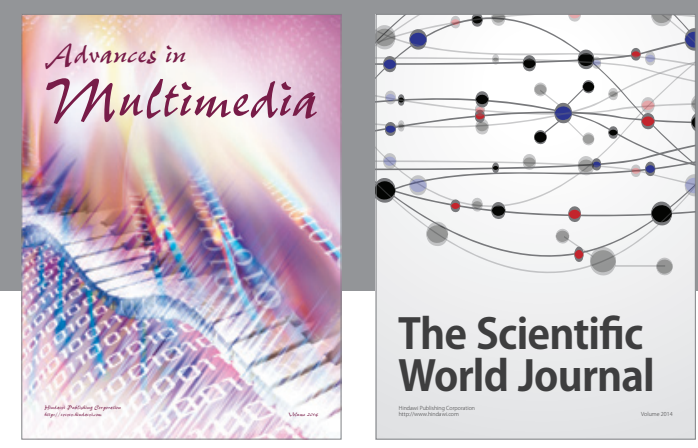

The Scientific World Journal
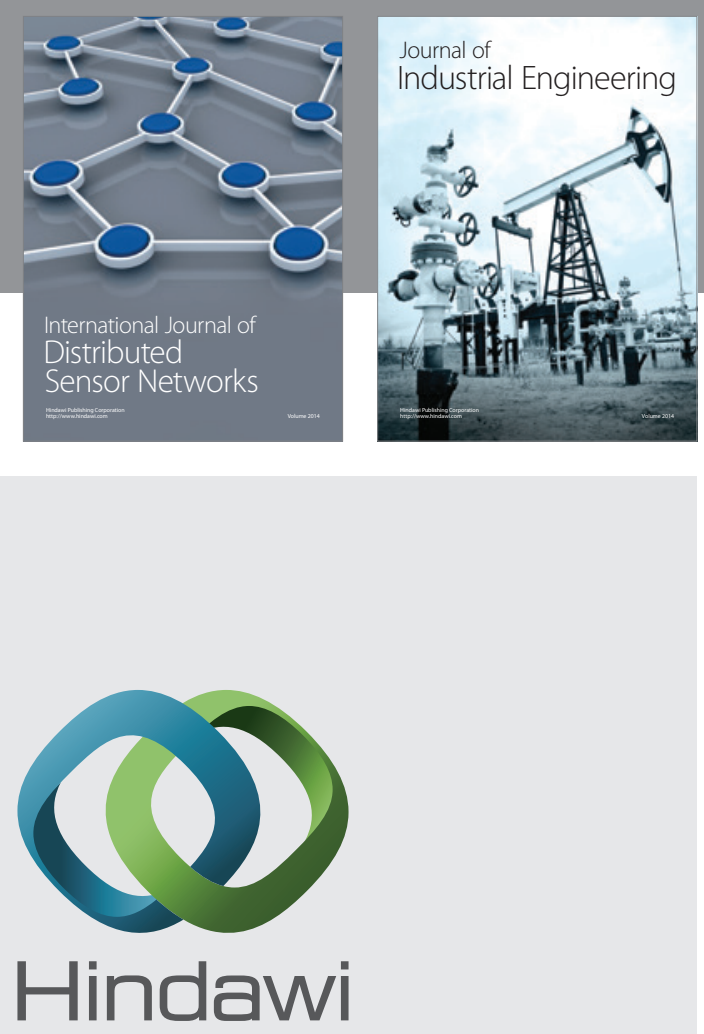

Submit your manuscripts at

https://www.hindawi.com

\section{Computer Networks} and Communications
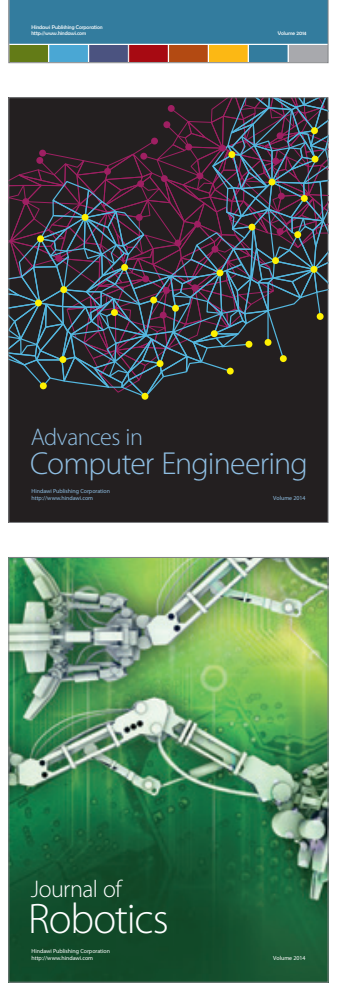
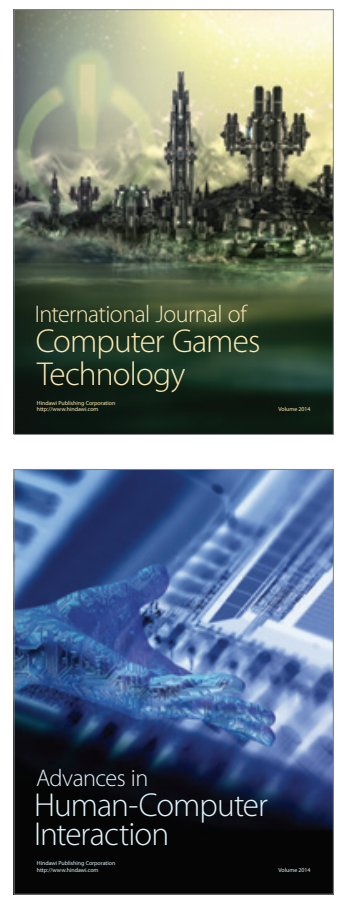
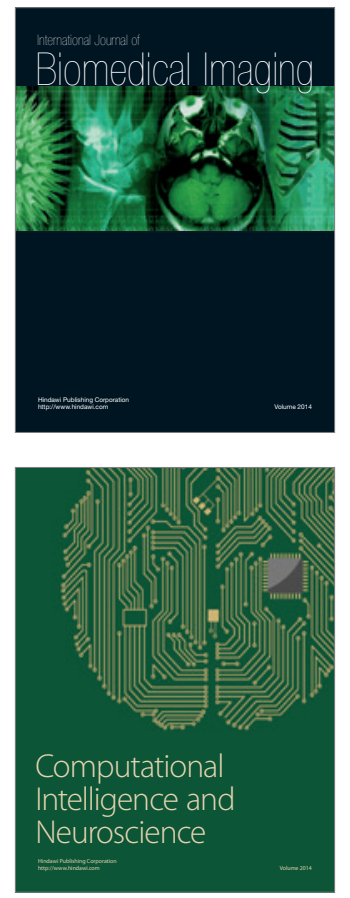
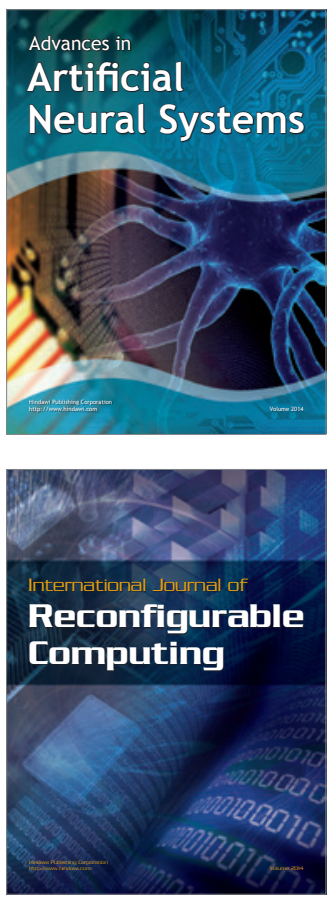
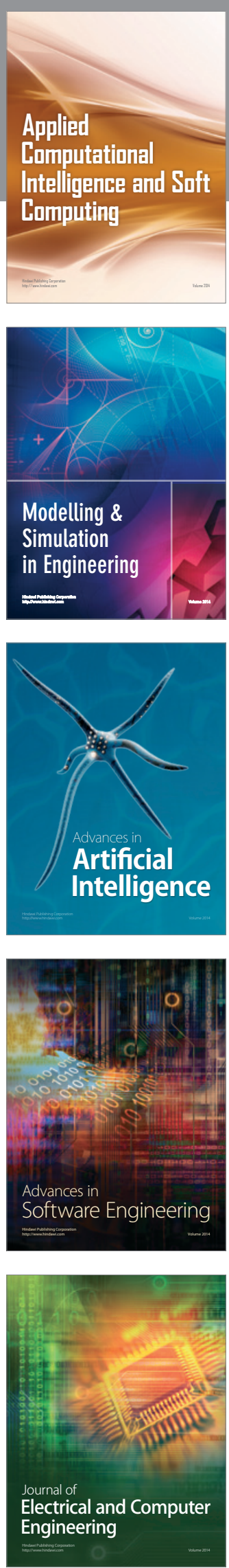